\title{
Relación entre Niveles de Inclusión de Terceros Molares Mandibulares e Índices Antropométricos Físicos de la Man- díbula en Etnias Atacameñas y Aymaras del Norte de Chile
}

\author{
Relationship between Levels of Third Molar Inclusion and Physical Anthropometric \\ Indices of the Mandible in the Atacameño and Aymara Ethnic Groups of Northern Chile
}

"Víctor Javier Beltrán Varas; *Ramón Fuentes Fernández; **Alejandro Bustos Cortés \& ****Antonio Sanhueza Campos

BELTRAN, V. V. J.; FUENTES, F. R.; BUSTOS, C. A.; SANHUEZA, C. A. Relación entre niveles de inclusión de terceros molares mandibulares e índices antropométricos físicos de la mandíbula en etnias Atacameñas y Aymaras del Norte de Chile. Int. J. Morphol., 29(2):446-454, 2011.

RESUMEN: La patología relacionada con el proceso de formación odontogénica, desarrollo y erupción de terceros molares, constituyen uno de los motivos más frecuentes de consulta odontológica. La mayoría de los autores coinciden en que el tercer molar inferior es el diente con mayor frecuencia de inclusión pero existen discrepancias respecto a la influencia de factores ecológicos y raciales que expliquen esa condición. Se sabe que los individuos de un grupo étnico tienen características físicas especiales y esto también se observa a nivel facial de acuerdo a las características del macizo máxilo-mandibular. Esto motivó investigar la relación entre niveles de inclusión de terceros molares mandibulares e índices antropométricos físicos de la mandíbula en grupos étnicos atacameños y aymaras del norte de Chile. De esta población se estableció una muestra de 56 pacientes para la determinación de grupos sanguíneos y factor Rh y establecer el grado de mestizaje. Asimismo se efectuó un examen clínico intra-oral para precisar el estado de erupción o ausencia de terceros molares inferiores y se tomaron ortopantomografías para determinar el estado de inclusión o agenesia de los dientes no visibles en la cavidad oral, a través de la clasificación de profundidad respecto del límite amelo-cementario del segundo molar presente. Finalmente, se procedió a registrar diferentes medidas del macizo máxilo-facial para establecer los índices de la rama bilateral e índice cigo-mandibular en cada uno de los individuos seleccionados. El estudio compara sus resultados con otras investigaciones y pudo determinar en ambas poblaciones indígenas la prevalencia de terceros molares inferiores $(85,72 \%$ derecha y $83,93 \%$ izquierda).

PALABRAS CLAVE: Antropología; Odontología; Tercer Molar; Inclusión.

\section{INTRODUCCIÓN}

La patología relacionada con el proceso de formación odontogénico, desarrollo y erupción de los terceros molares, constituyen uno de los motivos más frecuentes de consulta para el odontólogo (Albertos \& Junqueira, 2003; Susarla \& Dodson, 2004). Cuando dichas patologías aumentan en intensidad, generalmente se decide realizar la exodoncia de los terceros molares, en un procedimiento quirúrgico extendido en las piezas que presentan inclusión, que se ha estimado en alrededor de un $50 \%$ del costo de todos procedimientos quirúrgicos orales (Costas, 2003; Assael, 2005; Susarla \& Dodson, 2005).

El tercer molar inferior es el diente con mayor frecuencia de inclusión, influyendo factores ecológicos y ra- ciales entre otros (Susarla \& Dodson, 2005). Por consiguiente es importante determinar previamente en un paciente sus características antropométricas físicas del macizo máxilofacial, que son particulares en cada individuo, pero claramente relacionadas a aspectos raciales o étnicos que podrían determinar una condición previa más favorable o desfavorable al proceso de inclusión o retención de los terceros molares (Björk et al., 1956; Ricketts, 1972; Kaplan, 1974; Ventä et al., 2004; Better et al., 2004).

También se ha analizado, la asociación de diferentes niveles de inclusión de los terceros molares a ciertas edades como un método predictivo de erupción (Haug et al., 2005) y en relación a las clases esqueletales (Abu Alhaija et al., 2010).

\footnotetext{
* Departamento de Odontología Integral, Facultad de Medicina, Universidad de La Frontera, Chile.

*** Instituto de Investigaciones Antropológicas, Facultad de Educación y Ciencias Humanas, Universidad de Antofagasta, Chile.

**** Departamento de Matemáticas y Estadística, Facultad de Ingeniería, Universidad de La Frontera, Chile.
} 
Algunos autores han estudiado la asociación entre la presencia de terceros molares mandibulares y el origen de patologías a nivel de la mandíbula o estructuras neurovasculares relacionadas (Güven et al., 2000; Halmos et al., 2004; Akadiri et al., 2009; Inaoka et al., 2009; Ruga et al., 2010). Otros han investigado la asociación de la prevalencia del tercer molar mandibular con aspectos raciales o étnicos (Sandhu \& Kaur, 2005; García-Hernández \& Varas, 2008; García-Hernández \& Varas, 2009), encontrándose una prevalencia de terceros molares mandibulares, con diferencias en cuanto a los niveles de inclusión de $73,3 \%$ en la población atacameña, y 78,21\% en la aymará. La importancia de analizar ésta última asociación, tiene que ver con la incidencia epidemiológica del tercer molar como un ente con características especiales y una tendencia a desarrollar diferentes tipos de patologías en relación a las distintas poblaciones mundiales y también sus grupos étnicos.

En Chile, hay muy pocos estudios de prevalencia y etiopatogenia del tercer molar, menos aún en poblaciones étnicas específicas. Por esa razón esta investigación se enfocó, por una parte, en la relación entre profundidad de inclusión e índices mandibulares específicos en individuos de etnia atacameña y aymará, seleccionados por sus características antropométricas físicas de la mandíbula, especiales y similares entre sí; y por otra, dados los interesantes resultados obtenidos en estudios previos sobre la prevalencia de terceros molares (García-Hernández \& Varas, 2008, 2009).

El XVII Censo Nacional de Población y VI de Vivienda en abril de 2002 señala que la población en Chile es de 15116435 habitantes, de los cuales 692192 personas $(4,6 \%)$, dijeron pertenecer a uno de los ocho pueblos considerados en el instrumento Censal (Instituto Nacional de Estadísticas, Chile, 2002). La distribución de la población indígena chilena según su pertenencia étnica es muy heterogénea. En primer lugar, sobresale la elevada proporción del pueblo mapuche $(87,3 \%)$. Le siguen los aymara (7\%) y los atacameños (3\%) y otras etnias en menor proporción. Las etnias aymara y atacameñas se ubican en diversos territorios de norte del país, y aún cuando tienen características antropométricas similares, existe entre ellos diversidad cultural que se remontan desde épocas prehispánicas.

En las últimas cuatro décadas se intensificó una migración de las poblaciones indígenas desde las zonas rurales hacia los centros urbanos. En el caso de la migración aymara ésta se realizó en diferentes momentos y según áreas de proveniencia de los grupos migrantes lo que modificó la composición de las comunidades ubicadas en las zonas rurales del interior de la Región de Arica - Parinacota. En la actualidad dos tercios de los aymaras son mayoritariamente urbanos mientras que un tercio mantiene su condición rural.
De este modo surgen nuevos estilos de vida aymara en las ciudades lo que provoca procesos de adaptación cultural y social en estas nuevas comunidades indígenas urbanas que ya tiene dos generaciones. Estos movimientos migratorios se continúan produciendo hacia ciudades como Arica, Iquique, Pozo Almonte, Calama y Antofagasta, aumentando la incidencia del mestizaje biológico y cultural.

En el caso atacameño, hasta aproximadamente la década de 1950 las comunidades se ocupaban en actividades agrícolas, arriería, crianza de animales, etc. Pero también sus estilos de vida ya se encontraban fuertemente impactados y asimilados por la cultura nacional chilena. La condición de aislamiento de las comunidades de las zonas del Salar del Atacama y del Alto El Loa, junto con el impacto de la minería sobre los recursos naturales e hídricos, y la búsqueda de nuevas fuentes laborales incentivó de alguna manera fuertes procesos migratorios hacia las ciudades de la Región de Antofagasta como Chuquicamata y Calama.

Entre las demandas de las comunidades atacameñas destacan los derechos de las aguas, la constitución y saneamiento de las tierras bajo un criterio de coherencia territorial, reconocimiento y resguardo del territorio patrimonial y el reconocimiento, respeto y protección de la cultura atacameña. Los procesos migratorios también se han revertido existiendo un aumento de la población no atacameña y extranjera en San Pedro de Atacama, como producto de la dinámica de la actividad turística (Bustos, 1999; Bustos et al., 2007).

\section{MATERIAL Y MÉTODO}

Se desarrolló un estudio de corte transversal en diferentes situaciones resultantes de índices antropométricos físicos de la mandíbula y niveles de inclusión según profundidad de los terceros molares mandibulares en individuos indígenas con características antropométricas especiales de origen o ascendencia atacameña y aymara. La condición de indígena se estableció en base a los apellidos paterno y materno, certificados por la Corporación Nacional de Desarrollo Indígena (CONADI) y de acuerdo a consultas bibliográficas de la zona obtenida en la base de datos proporcionados por investigadores del Instituto de Investigaciones Antropológicas de la Universidad de Antofagasta.

Los individuos seleccionados en la muestra pertenecen a la comunas de Antofagasta, Calama, San Pedro de Atacama y Conchi Viejo y eran pacientes del Consultorio Norte de Antofagasta, Clínica dental Sindicato $\mathrm{N}^{\circ} 1$ de Calama, Hospital Militar del Norte, o también estudiantes 
de la Universidad de Antofagasta y Universidad Católica del Norte que integran la Agrupación Indígena Ayllu o Collasuyo de la Región de Antofagasta (Figs. 1 y 2).

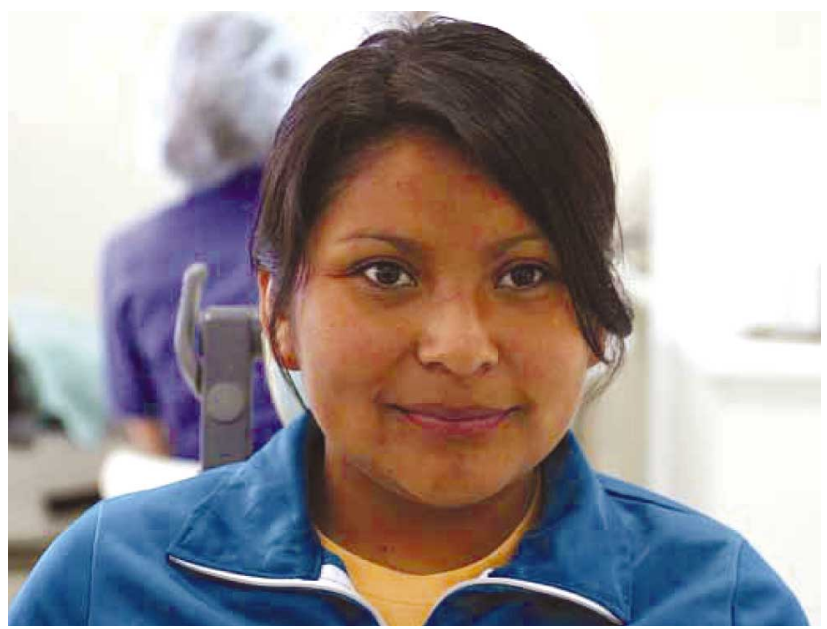

Fig. 1. Mujer Aymará que participó en el estudio.

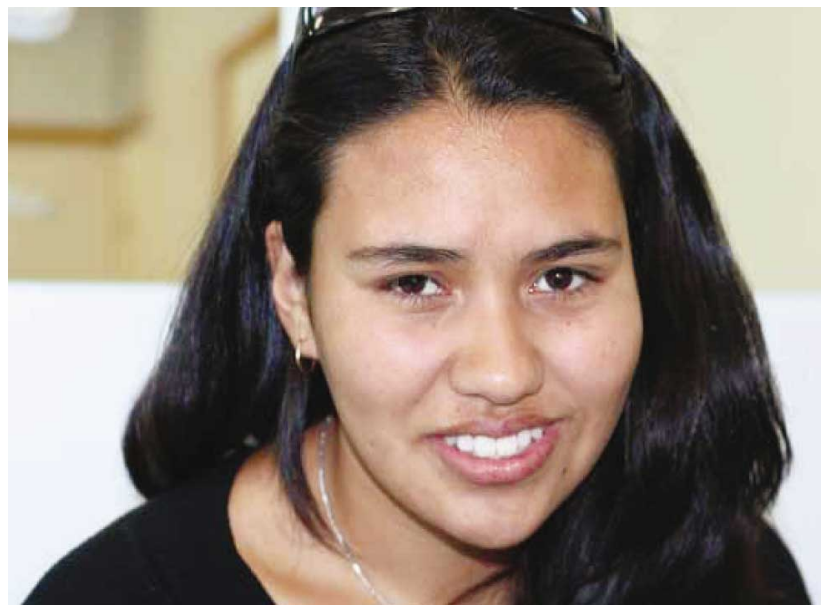

Fig. 2. Mujer Atacameña.

Asimismo se determinó el nivel de mestizaje de estas poblaciones indígenas, mediante la aplicación de la técnica serológica de hemoaglutinación con placas utilizando sueros testigos.

Con estos criterios se estableció una muestra de 56 personas de las cuales 30 (19 mujeres y 11 hombres) fueron de origen atacameño y 26 (12 mujeres y 14 hombres) de ascendencia aymará.

El grado de mestizaje de los individuos se determinó en base a la frecuencia génica de los alelos $\mathrm{O}$ y d (Rh-), del grupo sanguíneo y del factor $\mathrm{Rh}$, aplicando la fórmula de Bernstein: $\mathrm{M}=(\mathrm{Pm}-\mathrm{Pe}) /(\mathrm{Pi}-\mathrm{Pe})$, donde:

M: grado de mestizaje.
Pi: frecuencia génica en la población indígena.

Pe: frecuencia génica en la población española.

Pm: frecuencia génica en la población mixta.

La frecuencia génica de la población española fue realizada por Campillo, mientras que la frecuencia génica indioamericana fue comprobada por Matson et al. (ambos citados por Reyes, 2005), utilizando la fórmula de Bernstein.

En síntesis, se seleccionaron a individuos con las siguientes características:

1. De origen o ascendencia atacameña y aymará.

2. Entre 18 y 50 años.

3. No sometidos a exodoncia de terceros molares inferiores.

4. No sometidos a tratamiento de ortodoncia.

5. Con presencia del segundo molar permanente.

6. Sin antecedentes de trauma ni malformaciones máxilo-faciales.

Para el registro de esa información se elaboró una ficha clínica especialmente diseñada para efectos del presente estudio con debido consentimiento informado, en la cual fueron anotados nombre, sexo y edad, además de otros datos metodológicamente establecidos como un examen oral básico, registrando en la ficha los dientes presentes en boca y la localización de los terceros molares mandibulares (Figs. 3 y 4).

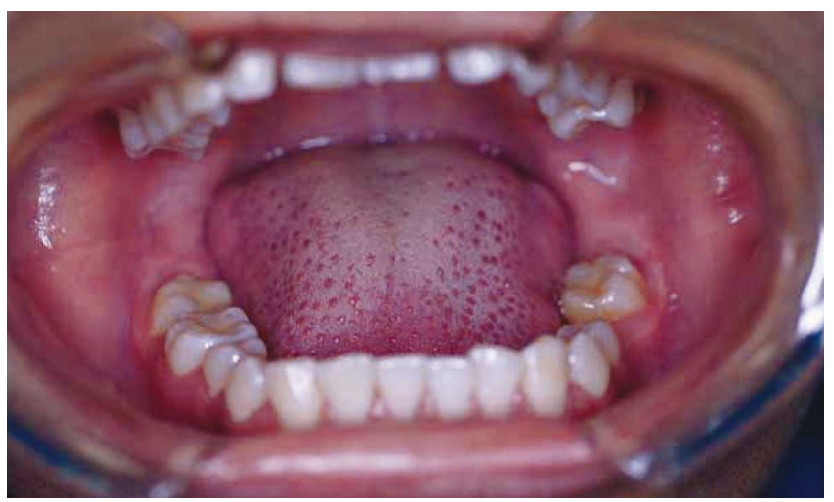

Fig. 3. Visión intraoral de individuo con inclusión de ambos terceros molares mandibulares.

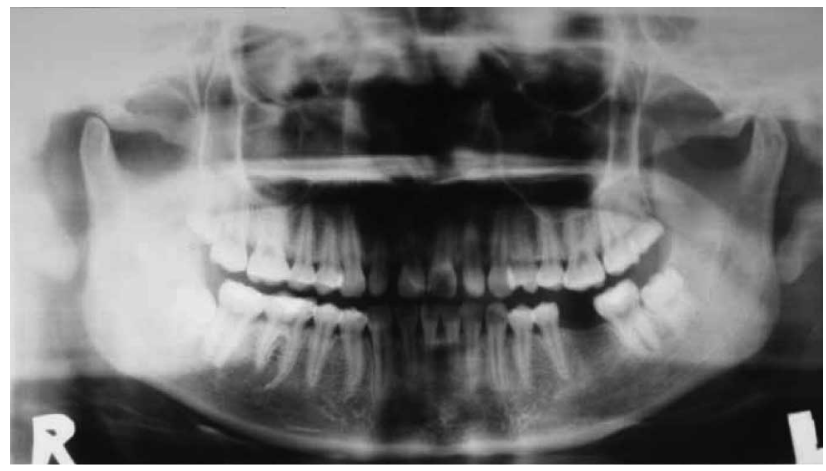

Fig. 4. Radiografía panorámica del mismo individuo. A través de ficha clínica diseñada para el estudio se registraban la presencia o ausencia intraoral de los terceros molares mandibulares y de estar presentes, se clasificaban según profundidad en sentido ápico-coronal respecto al límite amelo-cementario o cervical del segundo molar. 
Posteriormente se tomaron ortopantomografías (equipo radiográfico ARCOGRAPH ZEUS RF producido por IMAGO Radiology S.R.L. de Italia), a todos los individuos para analizar los niveles de inclusión según profundidad de los terceros molares mandibulares (Fig. 4). Se clasificaron los niveles de inclusión según la altura de la corona del tercer molar inferior con respecto plano de la unión coronoradicular o línea cervical del segundo molar inferior (Pell \& Gregory, 1933). Esta clasificación se describe de la siguiente manera (Fig. 5):

1. Clase A: La parte más alta de la corona del tercer molar esta al mismo nivel o sobre plano oclusal del segundo molar inferior (Fig. 5.1).

2. Clase B: La parte más alta de la corona del tercer molar esta bajo el plano oclusal, pero sobre el límite amelo-cementario o línea cervical del segundo molar (Fig. 5.2).

3. Clase C: La parte más alta de la corona del tercer molar está por debajo de la línea cervical del segundo molar (Fig. 5.3).
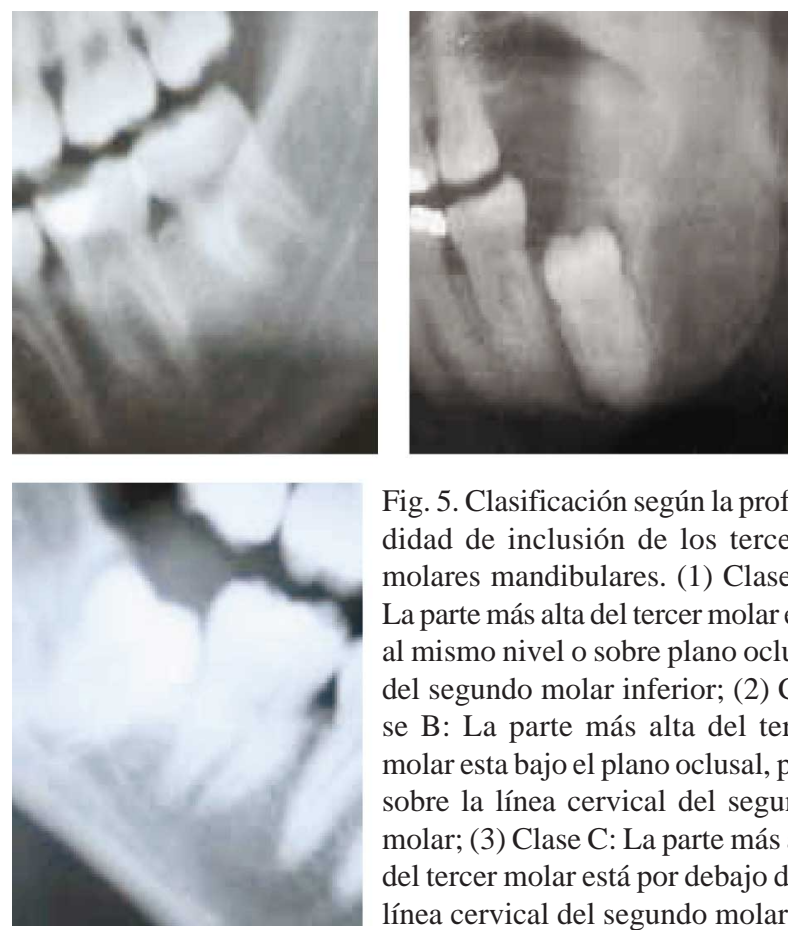

Fig. 5. Clasificación según la profundidad de inclusión de los terceros molares mandibulares. (1) Clase A: La parte más alta del tercer molar esta al mismo nivel o sobre plano oclusal del segundo molar inferior; (2) Clase B: La parte más alta del tercer molar esta bajo el plano oclusal, pero sobre la línea cervical del segundo molar; (3) Clase C: La parte más alta del tercer molar está por debajo de la línea cervical del segundo molar.

A continuación se realizaron las siguientes mediciones antropométricas físicas de la mandíbula. Para ello se utilizaron un craneómetro, pie de metro y compás de puntas romas. Las medidas se tomaron bilateralmente a excepción de los anchos bigonial y bicigomático por ser medidas únicas (Fig. 6, 7 y 8).
1. Longitud mandibular o distancia desde pogonion al borde posterior mandibular (Fig. 6.1 y 8.1).

2. Ancho mínimo de la rama mandibular, desde la parte más declive de la escotadura anterior de la rama mandibular al borde posterior de la rama, en una línea imaginaria paralela al piso (Fig. 6.2 y 8.2).

3. Ancho bigonial (Figs. 7.3 y 8.3).

4. Ancho bicigomático. (Figs. 7.4 y 8.4).

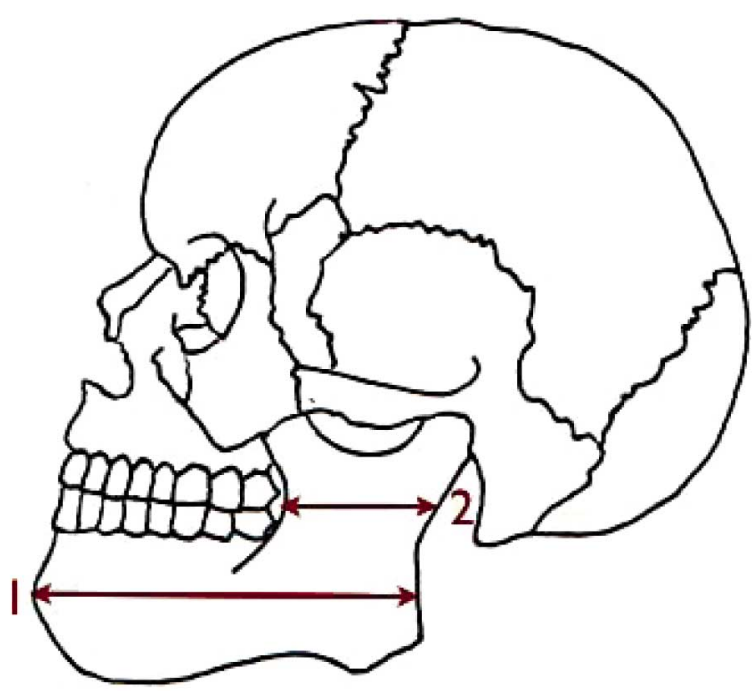

Fig. 6. Visión lateral que ilustra las medidas antropométricas físicas que fueron realizadas en el estudio. 1. Distancia desde pogonión al borde posterior mandibular; 2. Ancho mínimo de la rama mandibular.

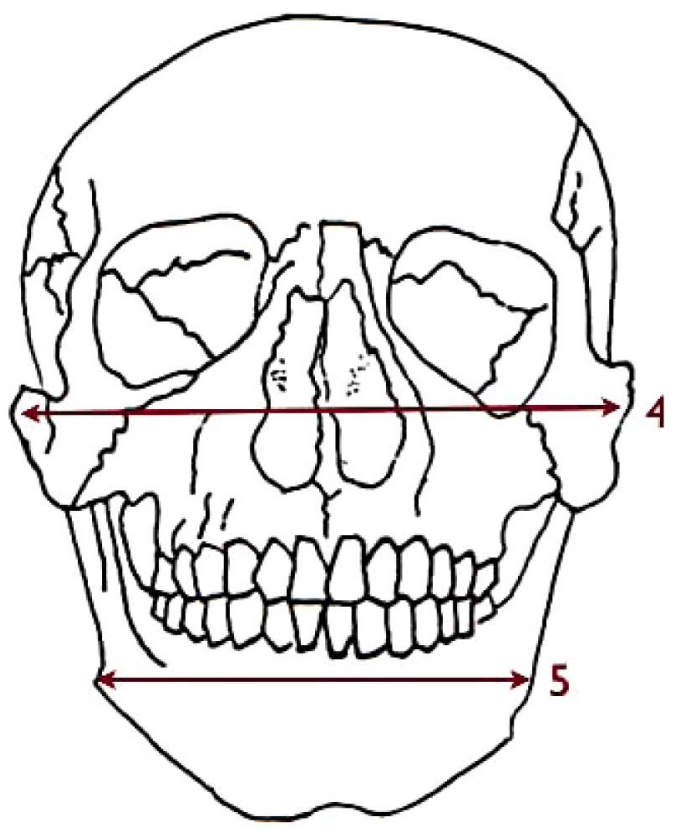

Fig. 7. Visión frontal que ilustra las medidas antropométricas físicas que fueron realizadas en el estudio. 4. Ancho bicigomático; 5. Ancho bigonial. 
Utilizando las mediciones obtenidas se calcularon los índices de la rama derecho e izquierdo, y el índice cigomático-mandibular descritos por Morel (1962) y Moya et al.(1994):

Anchura mínima de la rama:

Índice de la rama (IR):....... x 100

Longitud mandibular

Ancho bigonial

Índice cigo-mandibular (ICM):...... x 100

Ancho bicigomático

\section{RESULTADOS}

La distribución de los diferentes grupos sanguíneos se muestra en la Tabla I (ver Anexos). El grado de mestizaje de la muestra seleccionada fue superior al $50 \%$, para los individuos de ambas etnias (Tabla II).
La presencia de los terceros molares mandibulares fue de $85,72 \%$ derecha y $83,93 \%$ izquierda para ambos grupos indígenas.

Los niveles de inclusión resultantes de los terceros molares mandibulares según lado, en todos los individuos de ambas etnias se describen en la Tabla III, encontrándose una mayor frecuencia para la inclusión semi-profunda o tipo B, en ambos lados, seguida de la erupción total o tipo A, y finalmente la inclusión profunda o tipo $\mathrm{C}$ fue la menos prevalente.

Los índices antropométricos mandibulares para los individuos de atacameños y aymaras se describen en la Tabla IV. El promedio del índice de la rama derecha fue de $66,86 \%$ y de $68,10 \%$ para la izquierda.

El promedio del índice cigo-mandibular (85,8\%), es compatible con una mandíbula media para ambas poblaciones indígenas. Encontramos diferencias estadísticamente significativas (valor p igual a 0,046), en relación a inclusión profunda e índice cigo-mandibular de las dos etnias, siendo menor el índice cigo-mandibular a medida que la inclusión era más profunda (Tabla V).
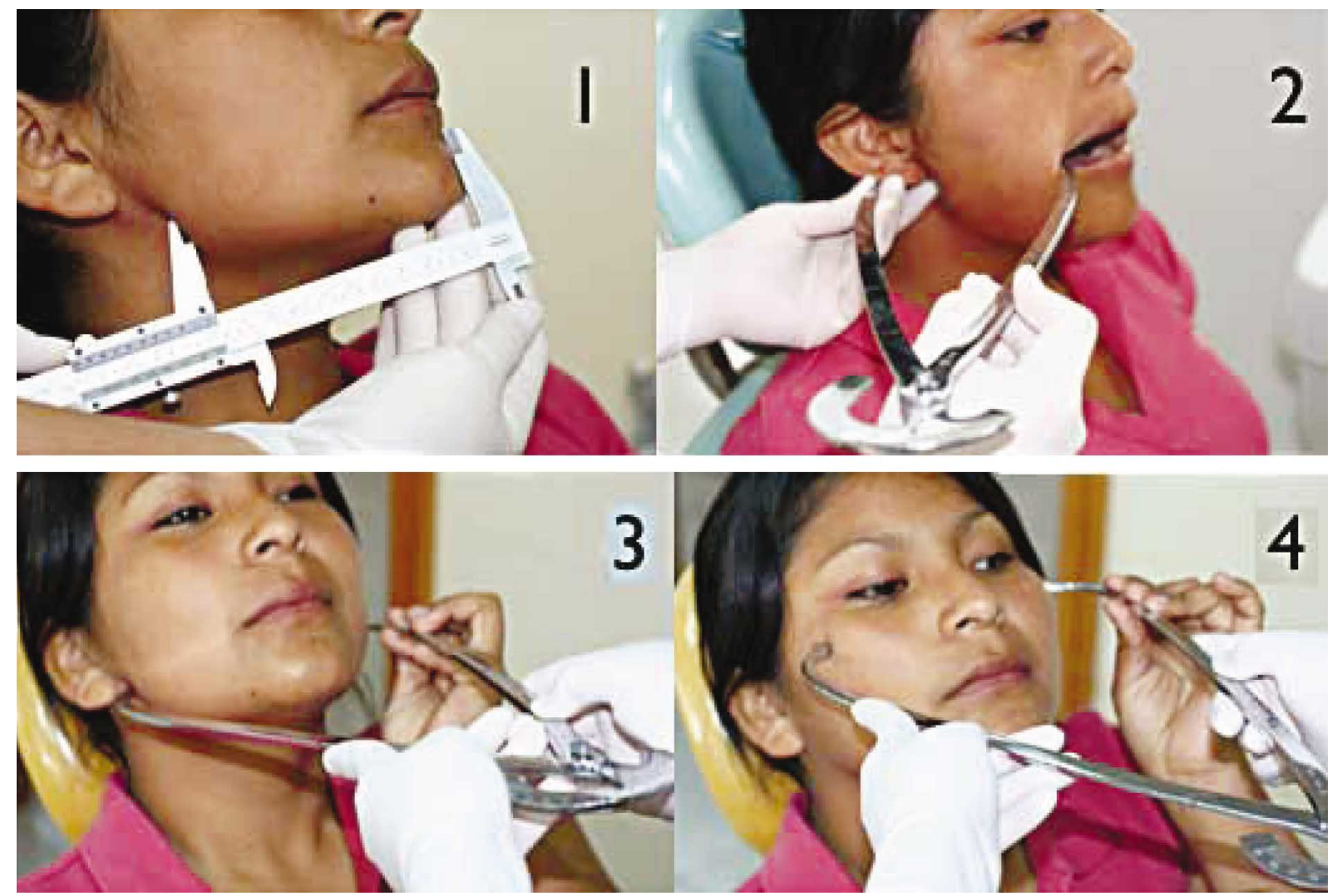

Fig. 8. Medidas antropométricas físicas en una mujer de ascendencia aymará. Se utilizaron regla milimetrada calibrada y craneómetro. 1. Distancia desde pogonión al borde posterior mandibular; 2. Ancho mínimo de la rama mandibular; 3 . Ancho bigonial; 4. Ancho bicigomático. 
Al relacionar el índice de la rama derecha e izquierda con los niveles de inclusión de los terceros molares inferiores, observamos una tendencia a un mayor índice de la rama izquierda a medida que la inclusión es más profunda o tipo C, aunque no se encuentra significancia estadística (Tabla VI).

Tabla I. Distribución de grupos sanguíneos detectados a través de técnica serológica de hemaglutinación por placas en la población estudio atacameña y aymara.

\begin{tabular}{lcr}
\hline Grupo Sanguíneo & Frecuencia & \multicolumn{1}{c}{$\%$} \\
\hline $\mathrm{A}+$ & 10 & 17,86 \\
$\mathrm{AB}+$ & 1 & 1,79 \\
$\mathrm{~B}+$ & 1 & 1,79 \\
$\mathrm{O}+$ & 41 & 73,21 \\
$\mathrm{O}-$ & 3 & 5,36 \\
\hline
\end{tabular}

Tabla II. Distribución del grado de mezcla indígena resultante en ambas etnias.

\begin{tabular}{lccc}
\hline & \multicolumn{2}{c}{ Frecuencia génica } & Mezcla indígena \\
\cline { 2 - 4 } & $\mathrm{O}$ & $\mathrm{D}(\mathrm{Rh}-)$ & $\%$ \\
Atacameños & 0,586 & 0,183 & 56 \\
Aymaras & 0,899 & 0,277 & 51 \\
\hline
\end{tabular}

Tabla III. Representación general de los diferentes niveles de inclusión de los terceros molares inferiores según lado (0: El molar no existe o es agenésico; A: La parte más alta del tercer molar esta al mismo nivel o sobre el plano oclusal del segundo molar inferior; B: La parte más alta del tercer molar esta bajo el plano oclusal pero sobre la línea cervical del segundo molar; $\mathrm{C}$ : La parte más alta del tercer molar esta por debajo de la línea cervical del segundo molar).

\begin{tabular}{lccc}
\hline Nivel de Inclusión & & Frecuencia & $\%$ \\
\hline \multirow{3}{*}{ ABC Derecho } & 0 & 8 & 14,29 \\
& $\mathrm{~A}$ & 17 & 30,36 \\
& $\mathrm{~B}$ & 30 & 53,57 \\
& $\mathrm{C}$ & 1 & 1,79 \\
\hline \multirow{3}{*}{ ABC Izquierdo } & 0 & 9 & 16,07 \\
& $\mathrm{~A}$ & 18 & 32,14 \\
& $\mathrm{~B}$ & 26 & 46,43 \\
& $\mathrm{C}$ & 3 & 5,36 \\
\hline
\end{tabular}

Tabla IV. Distribución general del número de individuos, promedios y desviación estándar de los diferentes índices antropométricos físicos de la mandíbula en todos los individuos (ambas etnias). (IRD: Índice de la rama mandibular derecha; IRI: Índice de la rama mandibular izquierda; ICM: Índice cigomático-mandibular).

\begin{tabular}{lcccc}
\hline $\begin{array}{l}\text { Índices } \\
\text { rama }\end{array}$ & $\begin{array}{c}\mathrm{n} \text { (Total de } \\
\text { individuos) }\end{array}$ & Promedio & D. E. \\
\hline IRD & 56 & 66,86 & 10,18 \\
IRI & 56 & 68,10 & 10,73 \\
ICM & 56 & 85,82 & 6,44 \\
\hline
\end{tabular}

Tabla V. Asociación global entre el promedio del índice cigo-mandibular y los niveles de inclusión según profundidad en todos los individuos (ambas etnias). Se observa asociación estadísticamente significativa en todos los individuos que presentaron inclusión profunda.

\begin{tabular}{lccccc}
\hline ABC lzquierdo & n Obs & Prome dio ICM & Mínimo & Máximo & Valor de p \\
\hline 0 & 9 & 86,80 & 78,57 & 96,15 & 0,046 \\
A & 18 & 87,20 & 76,92 & 104,50 & \\
B & 26 & 85,65 & 76,92 & 95,45 & \\
C & 3 & 76,17 & 61,87 & 83,33 & \\
\hline
\end{tabular}

Tabla VI. Asociación global entre el promedio del índice de la rama mandibular izquierda y derecha, y los niveles de inclusión izquierdo y derecho según profundidad en todos los individuos (ambas etnias). No se observa asociación estadísticamente significativa en ningún nivel de inclusión según profundidad.

\begin{tabular}{|c|c|c|c|c|c|c|c|c|c|c|c|}
\hline $\begin{array}{l}\text { ABC } \\
\text { Derecho }\end{array}$ & $\mathrm{n}$ & $\begin{array}{l}\text { Promedio } \\
\text { IRD }\end{array}$ & $\begin{array}{l}\text { Mínimo } \\
\text { IRD }\end{array}$ & $\begin{array}{l}\text { Máximo } \\
\text { IRD }\end{array}$ & $\begin{array}{l}\text { Valor } \\
\text { de p }\end{array}$ & $\begin{array}{c}\mathrm{ABC} \\
\text { Izquierdo }\end{array}$ & $\mathrm{n}$ & $\begin{array}{c}\text { Promedio } \\
\text { IRI }\end{array}$ & $\begin{array}{c}\text { Mínimo } \\
\text { IRI }\end{array}$ & $\begin{array}{c}\text { Máximo } \\
\text { IRI }\end{array}$ & $\begin{array}{c}\text { Valor de } \\
\text { p }\end{array}$ \\
\hline 0 & 8 & 70,82 & 61,40 & 95,23 & & 0 & 9 & 71,34 & 53,61 & 100 & \\
\hline A & 17 & 66,75 & 52,63 & 89,24 & & A & 18 & 67,37 & 52,85 & 78,39 & 0,110 \\
\hline B & 30 & 65,92 & 49,23 & 49,23 & & B & 26 & 66,03 & 46,87 & 86,06 & \\
\hline $\mathrm{C}$ & 1 & 65,35 & 65,35 & 65,35 & 0,695 & $\mathrm{C}$ & 3 & 80,66 & 67,32 & 98,33 & \\
\hline
\end{tabular}




\section{DISCUSIÓN}

El análisis de la información estadística existente en el sistema nacional de servicios de salud en Chile (S.N.S.S.), permite señalar que, en salud oral, las causas más frecuentes de consulta por urgencia son debidas, entre otras a: cuadros infecciosos consecutivos a caries dental; enfermedades periodontales y sus complicaciones; alteraciones en la erupción dentaria (dentro de la cual se presenta la mayoría de casos de exodoncias de terceros molares) y traumatismos dento-alveolares (Norma técnica de urgencia odontológica, 2003).

La mayoría de los autores coinciden que el tercer molar inferior es el diente con mayor frecuencia de inclusión, ocupando el canino superior el segundo lugar, influyendo factores ecológicos y raciales (Kaplan).

El estudio pudo determinar la prevalencia de terceros molares inferiores $(85,72 \%$ derecha y $83,93 \%$ izquierda), levemente superior a lo reportado en investigaciones previas (García-Hernández \& Varas, 2008; 2009), cuya diferencia puede deberse a que este estudio fue enfocado exclusivamente en terceros molares mandibulares.

La mayor frecuencia de inclusión corresponde al tipo B o semiprofunda, con un 53,57\% para el lado derecho y un 46,43\% para el lado izquierdo (Tabla III). Estos resultados difieren de lo reportado por Dias-Ribeiro et al. (2009) que encontraron la mayor frecuencia para el tipo A $(65,39 \%$ derecho, $51,85 \%$ izquierdo); pero concuerda con lo informado por Sandhu \& Kaur (2005), de una mayor prevalencia de la posición B o semiprofunda con un promedio de $39 \%$.

En relación a la inclusión profunda o tipo C, los hallazgos (1,79\% derecho, 5,36\% izquierdo) son similares a los de Dias-Ribeiro et al. (2,77\% derecho 3,03\% izquierdo) en ser la menos prevalente. Sin embargo difieren con Sandhu \& Kaur quienes reportaron un promedio de $34 \%$ de inclusión tipo $\mathrm{C}$, lo que podría deberse a las diferencias étnicas de los grupos estudiados.

Para los índices de la rama derecha e izquierda (66,86 y $68,10 \%$ respectivamente), éstos son ligeramente mayores que los determinados para la raza negra y mongoloides (entre 56 y 63\%), descritos por Moya et al.

El promedio del índice cigo-mandibular para los individuos atacameños y aymaras fue de 85,82\% (Tabla IV), lo que es coincidente con un mayor ensanchamiento del contorno facial desde la mandíbula al hueso cigomático, similar a caras mongoloides (Morel). Ello refleja una tenden- cia que se identificó clínicamente, pues estos individuos presentarían ramas mandibulares más anchas en sentido mesiodistal y una angulación más acentuada de ambas ramas en relación al ancho bicigomático, distinguiéndose como caras más redondas o mesoprosopas. Además se observó una tendencia a la inclusión profunda cuando índice cigomandibular es menor (Tabla V).

Sería interesante realizar un seguimiento de los casos de los terceros molares que presenten patologías, en un diseño de casos y controles y eventualmente diseños tipo cohortes concurrentes para pacientes adolescentes con características étnicas particulares hasta su adultez temprana.

Se debe determinar estas frecuencias de acuerdo al componente genético chileno y de las principales etnias que habitan tanto en el norte como en el sur de Chile, así como el entorno ecológico de la población seleccionada, de cuya frecuencia hay muy pocos antecedentes reportados.

Las etnias aymara y atacameñas se ubican en las regiones de Arica - Parinacota, Tarapacá, donde los factores ecológicos influyen de manera distinta en los estilos de vida de estos tipos de poblaciones indígenas ya sea si éstas habitan en los pueblos o aldeas rurales como las de San Pedro de Atacama o si residen en las grandes ciudades como Arica, Iquique, Calama y Antofagasta, a las cuales llegaron en calidad de emigrantes desde hace varias décadas desde sus poblados originarios, generándose entonces un fuerte mestizaje biológico y cultural (Bustos; Bustos et al.).

BELTRAN, V. V. J.; FUENTES, F. R.; BUSTOS, C. A.; SANHUEZA, C. A. Relationship between levels of third molar inclusion and physical anthropometric indices of the mandible in the Atacameño and Aymara ethnic groups of Northern Chile.Int. J. Morphol., 29(2):446-454, 2011.

SUMMARY: The pathology associated with the formation, development and eruption of third molars is one of the most frequent reasons for dental consults. Several authors agree that the third molar is the most frequent inclusion tooth, there is however, disagreement about the influence of environmental and racial factors that explain this condition. It is known that individuals of one ethnic group have particular physical characteristics, which are also observed at facial level according to characteristics of maxillo-mandibular mass. This prompted research regarding the relationship between levels of third molar inclusion and physical anthropometric indices of the mandible in Atacameño and Aymara ethnic groups in Northern Chile. A sample of 56 patients to determine blood type and $\mathrm{Rh}$ factor was established to assess the degree of mixing. An intra-oral clinical examination was also performed 
BELTRAN, V. V. J.; FUENTES, F. R.; BUSTOS, C. A.; SANHUEZA, C. A. Relación entre niveles de inclusión de terceros molares mandibulares e índices antropométricos físicos de la mandíbula en etnias Atacameñas y Aymaras del Norte de Chile. Int. J. Morphol., 29(2):446-454, 2011.

to clarify the state of eruption or absence of third molars. Radiographs were taken to determine the inclusion or agenesis if teeth were not visible in the oral cavity, through classification of depth on cementum-enamel boundary of the second molar present. Finally we proceeded to record various measurements of maxillofacial massif to set the bilateral mandibular ramus and ziggo mandibular indexes in each of the selected individuals. The study compares results with other research and determined in both indigenous populations, the prevalence of third molars $(85.72 \%$ right, and $83.93 \%$ left).

Inclusion.

KEY WORDS: Anthropology; Dentistry; Third Molar;

\section{REFERENCIAS BIBLIOGRÁFICAS}

Abu Alhaija, E. S.; Albhairan, H. M. \& Alkhateeb, S. N. Mandibular third molar space in different antero-posterior skeletal patterns. Eur. J. Orthod., 2010. doi: 10.1093/ ejo/cjq125

Akadiri, O. A.; Fasola, A. O. \& Arotiba, J. T. Incidence and risk factors for nerve injures in mandibular third molar surgery. Niger. J. Med., 18(4):402-8, 2009.

Albertos, C. J. M. \& Junqueira, G. L. M. Exodoncia selectiva de los terceros molares. Rev. Esp. Cir. Oral Maxilofac., 25(1):9-15, 2003.

Assael, L. A. Indications for elective therapeutic third molar removal: the evidence is in. J. Oral Maxillofac. Surg., 63(12):1691-2, 2005.

Better, H.; Abramovitz, I.; Shlomi, B.; Kahn, A.; Levy, Y., Shaham, A. \& Chaushu, G. The presurgical workup before third molar surgery: how much is enough? J. Oral Maxillofac. Surg., 62(6):689-92, 2004.

Björk, A.; Jensen, E. \& Palling, M. Mandibular growth and third molar impaction. Acta Odontol. Scand., 14:231-6, 1956.

Bustos, A. Etnografía Atacameña. $1^{\text {a }}$ ed. Antofagasta, Editorial Universidad de Antofagasta, 1999. pp.57-92.

Bustos, A.; Donoso, S. \& Fuentes, V. Construcción de una línea base sociocultural para el pueblo atacameño (Antofagasta, Chile). Revista Hontanar, IX(1):7-39, 2007.

Costas, L. A. Exodoncia rutinaria de terceros molares. Rev. Esp. Cir. Oral Maxilofac., 25(1):17-20, 2003.
Dias-Ribeiro, E.; Lima-Júnior, J. L.; Barbosa, J. L.; Barreto, H. I. \& Barbosa, S. L. L. Prevalencia de la posición de terceros molares inferiores retenidos con relación a la clasificación de Pell \& Gregory. Rev. Odontol. Mex., 13(4):229-33, 2009.

García-Hernández, F. \& Varas, V. J. B. Third Molar Agenesis in Native Ethnia from North of Chile: Atacameños or Lican Antai. Int. J. Morphol., 26(3):583-90, 2008.

García-Hernández, F. \& Varas, V. J. B. Third Molar Agenesis in Native Ethnia from North of Chile: Aymaras. Int. J. Morphol., 27(1):151-8, 2009.

Güven, O.; Keskin, A. \& Akal, U. K. The incidence of cysts and tumors around impacted third molars. Int. J. Oral Maxillofac. Surg., 29(2):131-5, 2000.

Halmos, D. R.; Ellis, E. 3rd. \& Dodson, T. B. Mandibular third molars and angle fractures. J. Oral Maxillofac. Surg., 62(9):1076-81, 2004.

Haug, R. H.; Perrott, D. H.; Gonzalez, M. L. \& Talwar, R. M. The American Association of Oral and Maxillofacial Surgeons Age-Related Third Molar Study. J. Oral Maxillofac. Surg., 63(8):1106-14, 2005.

Inaoka, S. D.; Carneiro, S. C.; Vasconcelos, B. C.; Leal, J. \& Porto, G. G. Relationship between mandibular fracture and impacted lower third molar. Med. Oral Patol. Oral Cir. Bucal, 14(7):E349-54, 2009.

Instituto Nacional de Estadísticas. Estadísticas Sociales de los Pueblos Indígenas de Chile Censo 2002, 2002. Disponible en: http://www.ine.cl/canales/chile_estadistico/ estadisticas_sociales_culturales/etnias/etnias.php

Kaplan, R. G. Mandibular third molars and postretention crowding. Am. J. Orthod., 66(4):411-30, 1974.

Moya, V.; Roldán, B. \& Sánchez, J. A. Odontología Legal y Forense. Barcelona, Masson, 1994.

Morel, P. Antropología Física. Buenos Aires, Universitaria, 1962.

Norma Técnica de urgencia Odontológica. Departamento de Salud Bucal, Ministerio de Salud, Gobierno de Chile, 2003. Disponible en: http:www.redsalud.gov.cl/.../ 7f2a6ebf9b5c1580e04001011e014d0e.pdf

Pell, G. J. \& Gregory, B. T. Impacted mandibular third molars: Classifications and modified techniques for removal. Dent. Digest., 39:330-8, 1933. 
BELTRAN, V. V. J.; FUENTES, F. R.; BUSTOS, C. A.; SANHUEZA, C. A. Relación entre niveles de inclusión de terceros molares mandibulares e índices antropométricos físicos de la mandíbula en etnias Atacameñas y Aymaras del Norte de Chile. Int. J. Morphol., 29(2):446-454, 2011.

Reyes, C. Parámetros de crecimiento facial en niños Mapuche. Santiago de Chile, Facultad de Odontología, Universidad de Chile, 2005.

Ricketts, R. A principle of racial growth of the mandible. Angle Orthod., 42(4):368-86, 1972.

Ruga, E.; Gallessio, C. \& Boffano, P. Mandibular alveolar neurovascular bundle injury associated with impacted third molar surgery. J. Craneofac. Surg., 21(4):1175-7, 2010 .

Sandhu, S. \& Kaur, T. Radiographic evaluation of the status of third molars in the Asian-Indian students. J. Oral Maxillofac. Surg., 63(5):640-5, 2005.

Susarla, S. M. \& Dodson, T. B. Risk factors for third molar extraction difficulty. J. Oral Maxillofac. Surg., 62(11):1363-71, 2004.

Susarla, S. M. \& Dodson, T. B. Howl well do clinicians estimate third molar extraction. J. Oral Maxillofac. Surg., 63(2):191-9, 2005.

Ventä, I.; Ylipaavalniemi, P. \& Turtola, L. Clinical outcome of third molars in adults followed during 18 years. $J$. Oral Maxillofac. Surg., 62(2):182-5, 2004.
Dirección para correspondencia:

Víctor Javier Beltrán Varas

Dirección Dpto. Odontología Integral

Facultad de Medicina

Calle Claro Solar № 115 -Of. 420

Universidad de La Frontera

TEMUCO

Email: vbeltranvaras@gmail.com

Recibido : 22-01-2011

Aceptado: 11-03-2011 